\title{
ON THE SATURATION EFFECT FOR LINEAR SHAPE-PRESERVING APPROXIMATION IN SOBOLEV SPACES
}

\author{
S. P. SIDOROV
}

Received 20 September, 2014

\begin{abstract}
We show that if a linear finite-dimensional operator defined in Sobolev space preserves $k$-monotonicity then the error of approximation of the operator does not decrease with the increase of smoothness of approximated functions. In other words, there is a saturation effect for linear finite-rank operators defined in Sobolev space and preserving $k$-monotonicity.
\end{abstract}

2010 Mathematics Subject Classification: 41A15; 65D17

Keywords: shape-preserving approximation, saturation effect, spline approximation

\section{INTRODUCTION}

In various applications of computer-aided geometric design it is necessary to approximate functions preserving such properties as monotonicity, convexity, concavity, etc. The part of the approximation theory, which is devoted to these kind of problems, is known as the theory of shape preserving approximation (see e.g. [3,9] for a survey).

A continuous function $f:[0,1] \rightarrow \mathbb{R}$ is said to be $k$-monotone, $k \geq 1$, on $[0,1]$ if and only if for all choices of $k+1$ distinct $t_{0}, \ldots, t_{k}$ in $[0,1]$ the inequality $\left[t_{0}, \ldots, t_{k}\right] f \geq 0$ holds, where $\left[t_{0}, \ldots, t_{k}\right] f$ denotes the $k$-th divided difference of $f$ at $0 \leq t_{0}<t_{1}<\ldots<t_{k} \leq 1$. Note that 2-monotone functions are just convex functions.

Let $\Delta^{k}$ denote the set of all $k$-monotone functions defined on [0,1]. If $f$ is a real-valued and $k$-times continuously differentiable function defined on $[0,1]$, then $f \in \Delta^{k}$ iff $f^{(k)}(t) \geq 0, t \in[0,1]$.

Let $W_{\infty}^{(k)}[0,1]$ be the Sobolev space of all real-valued, $(k-1)$-times differentiable functions whose derivative of order $(k-1)$ is absolutely continuous and whose

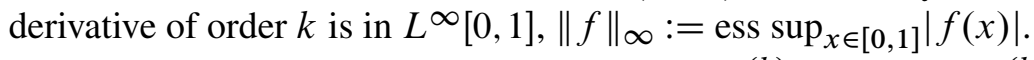

It is said that a linear operator $L$ of $W_{\infty}^{(k)}[0,1]$ into $W_{\infty}^{(k)}[0,1]$ preserves $k$-monotonicity, if $L\left(\Delta^{k}\right) \subset \Delta^{k}$.

The author was supported in part by Russian Ministry of Education and Science (project $1.1520 .2014 \mathrm{~K})$. 
Recall that a linear operator mapping a linear normed space into a linear space of finite dimension $n$ is called an operator of finite rank $n$.

Let $D^{i}$ denote the $i$-th differential operator, $D^{i} f(x)=d^{i} f(x) / d x^{i}$, and $D^{0}=I$ is the identity operator. Denote $e_{k}(x)=x^{k}, k=1,2, \ldots$.

Denote $B_{\infty}^{(k)}:=\left\{W_{\infty}^{(k)}[0,1]:\left\|D^{k} f\right\|_{\infty} \leq 1\right\}$.

Let $k \geq 1$. The main goal of the paper is to estimate the asymptotic value of relative linear $n$-width

$$
\inf _{L_{n}\left(\Delta^{k}\right) \subset \Delta^{k}} \sup _{f \in B_{\infty}^{(k)}}\left\|f-L_{n} f\right\|_{\infty}
$$

where infimum is taken over all linear continuous operators $L_{n}$ of $W_{\infty}^{(k)}[0,1]$ into $W_{\infty}^{(k)}[0,1]$ of finite rank $n$, such that $L_{n}\left(\Delta^{k}\right) \subset \Delta^{k}$ and $D^{k} L_{n} e_{k}=D^{k} e_{k}$. Note that some estimates of shape-preserving $n$-widths have been obtained in papers $[4,7,8]$.

T. Popoviciu [12] proved in 1937 that Bernstein operators

$$
B_{n} f(x):=\sum_{i=0}^{n} f\left(\frac{i}{n}\right) C_{n}^{i} x^{i}(1-x)^{n-i}
$$

preserve $k$-monotonicity of approximated functions, i.e. if $f$ is $k$-monotone on [0,1], then $B_{n} f$ is $k$-monotone on $[0,1]$ as well. However, it is well known $[2,13]$ that the order of approximation by Bernstein operators is low. In this paper we present the example of $k$-monotonicity preserving operator with the optimal order of approximation $n^{-2}(k \geq 2)$.

\section{LEMMAS}

Lemma 1. Let $L: W_{\infty}^{(k)}[0,1] \rightarrow W_{\infty}^{(k)}[0,1]$ be such that

(1) $L\left(\Delta^{k}\right) \subset \Delta^{k}$;

(2) $D^{k} L e_{k}=D^{k} e_{k}$.

If $f \in W_{\infty}^{(k)}[0,1]$ satisfies $\left\|D^{k} f\right\|_{\infty} \leq 1$, then $\left\|D^{k} L f\right\|_{\infty} \leq 1$.

Proof. For every $f \in W_{\infty}^{(k)}[0,1]$ such that $\left\|D^{k} f\right\|_{\infty} \leq 1$, we have

$$
\left|D^{k} f(x)\right| \leq 1=\frac{1}{k !} D^{k} e_{k}(x)
$$

for almost all $x \in[0,1]$. Since $L\left(\Delta^{k}\right) \subset \Delta^{k}$, for almost all $x \in[0,1]$ the following inequality holds:

Therefore, we have

$$
\left|D^{k} L f(x)\right| \leq \frac{1}{k !} D^{k} L e_{k}(x) .
$$

$$
\left\|D^{k} L f\right\|_{\infty} \leq \frac{1}{k !}\left\|D^{k} L e_{k}\right\|_{\infty} .
$$

It follows from $D^{k} L e_{k}=D^{k} e_{k}$ that $\left\|D^{k} L f\right\|_{\infty} \leq 1$. 
Lemma 1 says that if $L: W_{\infty}^{(k)}[0,1] \rightarrow W_{\infty}^{(k)}[0,1]$ satisfies conditions (i) and (ii) then $L$ preserves the ball $B_{\infty}^{(k)}$, i.e. $L\left(B_{\infty}^{(k)}\right) \subset B_{\infty}^{(k)}$.

Denote $z_{j}:=j / n, j=0, \ldots, n$. Let the linear operator $M_{k, n}: W_{\infty}^{(k)}[0,1] \rightarrow$ $W_{\infty}^{(k)}[0,1], k \geqslant 2, n \geq 2$, be defined by

$$
\begin{aligned}
M_{k, n} f(x)= & \sum_{l=0}^{k-1} \frac{1}{l !} x^{l} D^{l} f(0)+\frac{2}{k !} x^{k}\left[z_{0}, z_{1}, z_{2}\right] D^{k-2} f \\
& +\frac{2 n}{(k+1) !} x^{k+1}\left(\left[z_{1}, z_{2}, z_{3}\right] D^{k-2} f-\left[z_{0}, z_{1}, z_{2}\right] D^{k-2} f\right),
\end{aligned}
$$

if $x \in\left[z_{0}, z_{1}\right]$;

$$
\begin{aligned}
M_{k, n} f(x)= & \sum_{l=0}^{k-1} \frac{1}{l !}\left(x-z_{j}\right)^{l} D^{l} M_{k, n} f\left(z_{j}\right) \\
& +\frac{2}{k !}\left(x-z_{j}\right)^{k}\left[z_{j}, z_{j+1}, z_{j+2}\right] D^{k-2} f+\frac{2 n}{(k+1) !}\left(x-z_{j}\right)^{k+1} \\
& \times\left(\left[z_{j+1}, z_{j+2}, z_{j+3}\right] D^{k-2} f-\left[z_{j}, z_{j+1}, z_{j+2}\right] D^{k-2} f\right), \\
\text { if } x & \in\left(z_{j}, z_{j+1}\right], j=1,2, \ldots, n-3 ;
\end{aligned}
$$

$M_{k, n} f(x)=\sum_{l=0}^{k-1} \frac{1}{l !}\left(x-z_{n-2}\right)^{l} D^{l} M_{k, n} f\left(z_{n-2}\right)$

$$
\begin{aligned}
& +\frac{2}{k !}\left(x-z_{n-2}\right)^{k}\left[z_{n-3}, z_{n-2}, z_{n-1}\right] D^{k-2} f+\frac{2 n}{(k+1) !}\left(x-z_{n-2}\right)^{k+1} \\
& \times\left(\left[z_{n-2}, z_{n-1}, z_{n}\right] D^{k-2} f-\left[z_{n-3}, z_{n-2}, z_{n-1}\right] D^{k-2} f\right)
\end{aligned}
$$

if $x \in\left(z_{n-2}, z_{n-1}\right]$;

$$
\begin{aligned}
M_{k, n} f(x)= & \sum_{l=0}^{k-1} \frac{1}{l !}\left(x-z_{n-1}\right)^{l} D^{l} M_{k, n} f\left(z_{n-1}\right) \\
& +\frac{2}{k !}\left(x-z_{n-1}\right)^{k}\left[z_{n-3}, z_{n-2}, z_{n-1}\right] D^{k-2} f+\frac{2 n}{(k+1) !}\left(x-z_{n-1}\right)^{k+1} \\
& \times\left(\left[z_{n-2}, z_{n-1}, z_{n}\right] D^{k-2} f-\left[z_{n-3}, z_{n-2}, z_{n-1}\right] D^{k-1} f\right), \\
\text { if } x & \in\left(z_{n-1}, 1\right] .
\end{aligned}
$$


Lemma 2. Let $k \geq 2$ and let the operator $M_{k, n}: W_{\infty}^{(k)}[0,1] \rightarrow W_{\infty}^{(k)}[0,1]$ be defined by (2.1), (2.2), (2.3), (2.4). Then

(1) $M_{k, n}\left(\Delta^{k}\right) \subset \Delta^{k}$;

(2) $D^{k} M_{k, n} e_{k}=D^{k} e_{k}$;

(3) there exists a real $c>0$ such that $\left\|f-M_{k, n} f\right\|_{\infty} \leq c n^{-2}$ for all $f \in B_{\infty}^{(k)}$.

Proof. Note that $D^{k} M_{k, n} f$ is a piecewise linear function and $D^{k} M_{k, n} f$ is linear on $\left[z_{j}, z_{j+1}\right]$ for each $0 \leq j \leq n-1$. If $x \in\left(z_{j}, z_{j+1}\right]$, then

$$
\begin{aligned}
D^{k} M_{k, n} f(x)=2 & (j+1-n x)\left[z_{j}, z_{j+1}, z_{j+2}\right] D^{k-2} f \\
+ & 2(n x-j)\left[z_{j+1}, z_{j+2}, z_{j+3}\right] D^{k-2} f, j=0,1, \ldots, n-3, \\
D^{k} M_{k, n} f(x)= & 2(n-1-n x) \cdot\left[z_{n-3}, z_{n-2}, z_{n-1}\right] D^{k-2} f \\
& +2(n x-n+2) \cdot\left[z_{n-2}, z_{n-1}, z_{n}\right] D^{k-2} f, j=n-2 . \\
D^{k} M_{k, n} f(x)= & 2(n-n x) \cdot\left[z_{n-3}, z_{n-2}, z_{n-1}\right] D^{k-2} f \\
& +2(n x-n+1) \cdot\left[z_{n-2}, z_{n-1}, z_{n}\right] D^{k-2} f, j=n-1 .
\end{aligned}
$$

If $f \in \Delta^{k}$, then it follows from (2.5), (2.6), (2.7) that $D^{k} M_{k, n} f \geq 0$ on each $\left(x_{j}, x_{j+1}\right), j=0, \ldots, n-1$, i.e. $M_{k, n} f \in \Delta^{k}$. Therefore, $M_{k, n}\left(\Delta^{k}\right) \subset \Delta^{k}$.

A direct verification shows that $D^{k} M_{k, n} e_{k}=D^{k} e_{k}$.

Since $f \in B_{\infty}^{(k)}$, the $(k-2)$-th derivative of $f$ can be represented as

$$
D^{k-2} f(x)=D^{k-2} f(0)+\frac{D^{k-1} f(0)}{1 !} x+\int_{0}^{1}(x-t)_{+} D^{k} f(t) d t,
$$

where $\left\|D^{k} f\right\|_{\infty} \leq 1$ and $y_{+}=y$ if $y \geq 0$, and 0 otherwise.

Take an arbitrary $x \in\left[0, \frac{1}{n}\right]$ (the case $x \in\left(z_{j}, z_{j+1}\right), j=1, \ldots, n-3$, can be examined analogously), then

$$
\begin{aligned}
& D^{k-2} M_{k, n} f(x)-D^{k-2} f(x) \\
&=x^{2}\left[z_{0}, z_{1}, z_{2}\right] D^{k-2} f+\frac{1}{3} n x^{3}\left(\left[z_{1}, z_{2}, z_{3}\right] D^{k-2} f-\left[z_{0}, z_{1}, z_{2}\right] D^{k-2} f\right) \\
&-\int_{0}^{1}(x-t)_{+} D^{k} f(t) d t \\
&= \int_{0}^{1}\left[x^{2}\left(\left(z_{0}-t\right)_{+}-2\left(z_{1}-t\right)_{+}+\left(z_{2}-t\right)_{+}\right)\right. \\
&+\frac{1}{3} n x^{3}\left(\left(z_{1}-t\right)_{+}-2\left(z_{2}-t\right)_{+}+\left(z_{3}-t\right)_{+}\right) \\
&\left.-\frac{1}{3} n x^{3}\left(\left(z_{0}-t\right)_{+}-2\left(z_{1}-t\right)_{+}+\left(z_{2}-t\right)_{+}\right)-(x-t)_{+}\right] D^{k} f d t,
\end{aligned}
$$


and we can obtain

$$
\left|D^{k-2} M_{k, n} f(x)-D^{k-2} f(x)\right| \leq a n^{-2}, x \in\left[z_{j}, z_{j+1}\right], j=1, \ldots, n-3,
$$

for some $a>0$. In the same way it can be shown that

$$
\left|D^{k-2} M_{k, n} f(x)-D^{k-2} f(x)\right| \leq n^{-2}, x \in\left[z_{n-2}, z_{n-1}\right], \text { and } x \in\left[z_{n-1}, 1\right] .
$$

Therefore, for every $f \in B_{\infty}^{(k)}$ and any $x \in[0,1]$

$$
\left|D^{k-2} f(x)-D^{k-2} M_{k, n} f(x)\right| \leq c_{1} n^{-2} .
$$

If $k=2$ then the proposition (3) of Lemma 2 is proved.

Let $k \geq 3$. Since $D^{i}\left(M_{k, n} f-f\right)(0)=0$ holds for $i=0, \ldots, k-3$, we have

$$
\begin{aligned}
D^{i}\left(M_{k, n} f-f\right)(x) & =D^{i}\left(M_{k, n} f-f\right)(0)+\int_{0}^{x} D^{i+1}\left(M_{k, n} f-f\right)(t) d t \\
& =\int_{0}^{x} D^{i+1}\left(M_{k, n} f-f\right)(t) d t
\end{aligned}
$$

for all $x \in[0,1]$. Then

$$
\left|D^{i}\left(M_{k, n} f-f\right)(x)\right| \leq \frac{1}{n^{2}} \frac{x^{k-1-i}}{(k-1-i) !} .
$$

We have used the fact that if $f \in L_{\infty}[0,1]$ and there exists a real $a \in \mathbb{R}$ such that $|f| \leq a$ on $[0,1]$, then

$$
\left|\int_{0}^{x} \int_{0}^{t_{p-1}} \ldots \int_{0}^{t_{1}} f\left(t_{1}\right) d t_{1} \ldots d t_{p}\right| \leq \int_{0}^{x} \int_{0}^{t_{p-1}} \ldots \int_{0}^{t_{1}}\left|f\left(t_{1}\right)\right| d t_{1} \ldots d t_{p} \leq a \frac{x^{p}}{p !}
$$

for every $p \in \mathbb{N}$.

The proposition (3) of Lemma 2 follows from (2.9) with $i=0$.

\section{THE MAIN RESULT}

Theorem 1. Let $k \in \mathbb{N}$. Then

$$
\inf _{L_{n}\left(\Delta^{k}\right) \subset \Delta^{k}} \sup _{f \in B_{\infty}^{(k)}}\left\|f-L_{n} f\right\|_{\infty} \asymp\left\{\begin{array}{l}
n^{-2}, k \geqslant 2, \\
n^{-1}, k=1,
\end{array}\right.
$$

where infimum is taken over all linear continuous operators $L_{n}$ of $W_{\infty}^{(k)}[0,1]$ into $W_{\infty}^{(k)}[0,1]$ of finite rank $n$, such that

(1) $L_{n}\left(\Delta^{k}\right) \subset \Delta^{k}$;

(2) $D^{k} L_{n} e_{k}=D^{k} e_{k}$. 
Proof. We have (by definition)

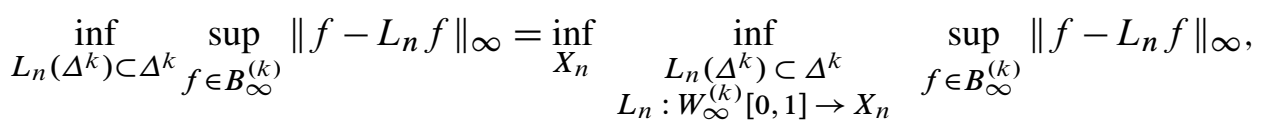

where the first infimum is taken over all $n$-dimensional subspaces $X_{n}$ of $W_{\infty}^{(k)}[0,1]$, and the second one is taken over all continuous linear operators $L_{n}$ of $W_{\infty}^{(k)}[0,1]$ into $X_{n}$ satisfying $L_{n}\left(\Delta^{k}\right) \subset \Delta^{k}$ and $D^{k} L_{n} e_{k}=D^{k} e_{k}$.

Since $f \in B_{\infty}^{(k)}$, it follows from Lemma 1 that $L_{n} f \in B_{\infty}^{(k)}$. Then Eq. (3.2) implies

$$
\inf _{L_{n}\left(\Delta^{k}\right) \subset \Delta^{k}} \sup _{f \in B_{\infty}^{(k)}}\left\|f-L_{n} f\right\|_{\infty} \geqslant \inf _{X_{n}} \sup _{f \in B_{\infty}^{(k)}} \inf _{g \in B_{\infty}^{(k)} \cap X_{n}}\|f-g\|_{\infty} .
$$

It was shown in the paper of Konovalov [6] that

$$
\inf _{X_{n}} \sup _{f \in B_{\infty}^{(k)}} \inf _{g \in B_{\infty}^{(k)} \cap X_{n}}\|f-g\|_{\infty} \asymp\left\{\begin{array}{l}
n^{-2}, k \geqslant 2, \\
n^{-1}, k=1 .
\end{array}\right.
$$

To complete the proof it is sufficient to show that there exists a linear finitedimensional method, that is optimal for $n$-widths (3.1). If $k \geqslant 2$, then as it follows from Lemma 2, the linear operator $M_{k, n}: W_{\infty}^{(k)}[0,1] \rightarrow W_{\infty}^{(k)}[0,1]$ satisfies the conditions of Theorem and it is optimal for $n$-width (3.1). If $k=1$, then the piecewise linear interpolation method with breakpoints $z_{j}, j=0, \ldots, n$, is optimal for $n$-width (3.1).

Theorem 1 shows that the shape-preserving property of operators is negative in the sense that the error of approximation of such operators does not decrease with the increase of smoothness of approximated functions. In other words, there is a saturation effect for linear finite-rank operators preserving $k$-monotonicity. For the first time the problem was mentioned by Ronald A. De Vore in his paper [1]. It is worth noting that non-linear approximation preserving $k$-monotonicity does not have this shortcoming [10]. On the other hand, for sequences of linear operators preserving $k$-monotonicity (as well as intersections of cones) there are $[5,11]$ simple convergence conditions (Korovkin type results).

\section{REFERENCES}

[1] R. A. De Vore, "Monotone approximation by splines," SIAM J. Math. Anal., vol. 8, pp. 891-905, 1977, doi: 10.1137/0508068.

[2] M. Floater, "On the convergence of derivatives of Bernstein approximation," Journal of Approximation Theory, vol. 134, no. 1, pp. 130 - 135, 2005, doi: 10.1016/j.jat.2004.02.009.

[3] S. G. Gal, Shape-Preserving Approximation by Real and Complex Polynomials. Springer, 2008.

[4] J. Gilewicz, V. N. Konovalov, and D. Leviatan, "Widths and shape-preserving widths of Sobolevtype classes of s-monotone functions," J. Approx. Theory, vol. 140, no. 2, pp. 101-126, 2006, doi: 10.1016/j.jat.2005.11.016. 
[5] H. H. Gonska, "Quantitative Korovkin type theorems on simultaneous approximation," Mathematische Zeitschrift, vol. 186, no. 3, pp. 419-433, 1984, doi: 10.1007/BF01174895.

[6] V. Konovalov, "Estimates of Kolmogorov type widths for classes of differentiable functions," Mat. Notes, vol. 35, no. 3, pp. 193-199, 1984, doi: 10.1007/BF01139916.

[7] V. Konovalov and D. Leviatan, "Shape preserving widths of Sobolev-type classes of $k$-monotone functions on a finite interval," Israel Journal of Mathematics, vol. 133, pp. 239-268, 2003, doi: 10.1007/BF02773069.

[8] V. Konovalov and D. Leviatan, "Shape-preserving widths of weighted Sobolev-type classes of positive, monotone, and convex functions on a finite interval," Constructive Approximation, vol. 19, pp. 23-58, 2003, doi: 10.1007/s00365-001-0027-3.

[9] K. A. Kopotun, D. Leviatan, A. Prymak, and I. A. Shevchuk, "Uniform and pointwise shape preserving approximation by algebraic polynomials," Surveys in Approximation Theory, vol. 6, pp. 24-74, 2011

[10] K. Kopotun and A. Shadrin, "On k-monotone approximation by free knot splines," SIAM J. Math. Anal., vol. 34, pp. 901-924, 2003, doi: 10.1137/S0036141002358514.

[11] F. J. Muñoz-Delgado, V. Ramírez-González, and D. Cárdenas-Morales, "Qualitative Korovkintype results on conservative approximation," J. Approx. Theory, vol. 94, pp. 144-159, 1998, doi: 10.1006/jath.1998.3182.

[12] T. Popoviciu, About the Best Polynomial Approximation of Continuous Functions. Mathematical Monography. Sect. Mat. Univ. Cluj, 1937, (In Romanian), fasc. III.

[13] E. Voronovskaya, "Determination de la forme asymptotique de l'approximation des fonctions par les polynomes de M. Bernstein,” Dokl. Akad. Nauk SSSR, vol. A, pp. 79-85, 1932.

Author's address

\section{S. P. Sidorov}

Department of Mechanics and Mathematics, Saratov State University, Saratov, Russian Federation E-mail address: sidorovsp@info.sgu.ru 